\title{
Myt3 suppression sensitizes islet cells to high glucose-induced cell death via Bim induction
}

\author{
BR Tennant ${ }^{1}$, B Vanderkruk ${ }^{1}$, J Dhillon ${ }^{1}$, D Dai ${ }^{1}$, CB Verchere ${ }^{1,2,3}$ and BG Hoffman ${ }^{\star, 1,2}$
}

Diabetes is a chronic disease that results from the body's inability to properly control circulating blood glucose levels. The loss of glucose homoeostasis can arise from a loss of $\beta$-cell mass because of immune-cell-mediated attack, as in type 1 diabetes, and/or from dysfunction of individual $\beta$-cells (in conjunction with target organ insulin resistance), as in type 2 diabetes. A better understanding of the transcriptional pathways regulating islet-cell survival is of great importance for the development of therapeutic strategies that target $\beta$-cells for diabetes. To this end, we previously identified the transcription factor Myt3 as a prosurvival factor in islets following acute suppression of Myt3 in vitro. To determine the effects of Myt3 suppression on islet-cell survival in vivo, we used an adenovirus to express an shRNA targeting Myt3 in syngeneic optimal and marginal mass islet transplants, and demonstrate that suppression of Myt3 impairs the function of marginal mass grafts. Analysis of grafts 5 weeks post-transplant revealed that grafts transduced with the shMyt3 adenovirus contained $\sim 20 \%$ the number of transduced cells as grafts transduced with a control adenovirus. In fact, increased apoptosis and significant cell loss in the shMyt3-transduced grafts was evident after only 5 days, suggesting that Myt3 suppression sensitizes islet cells to stresses present in the early posttransplant period. Specifically, we find that Myt3 suppression sensitizes islet cells to high glucose-induced cell death via upregulation of the pro-apoptotic Bcl2 family member Bim. Taken together these data suggest that Myt3 may be an important link between glucotoxic and immune signalling pathways.

Cell Death and Disease (2016) 7, e2233; doi:10.1038/cddis.2016.141; published online 19 May 2016

Type 1 diabetes (T1D) results from autoimmunity gradually leading to a loss of $\beta$-cells and hyperglycaemia. ${ }^{1}$ It is thought that recruitment and activation of auto-reactive $T$ cells targeting $\beta$-cells is the primary inflammatory process driving the disease. ${ }^{2-4}$ Recruited immune cells produce Fas, perforin, $\mathrm{IL}-1 \beta$, TNF $\alpha$ and IFN $\gamma$ that bind to receptors on the surface of $\beta$-cells leading to activation of downstream signalling cascades and inducing changes in $\beta$-cell gene expression resulting in the induction of $\beta$-cell dysfunction and apoptosis. ${ }^{5-10}$ It is essential that we develop strategies to halt the immune assault on the islets, and prevent cytokine-induced changes in $\beta$-cell gene expression and apoptosis. There remain, however, significant gaps in our understanding of the regulatory processes and downstream mediators of cytokineinduced $\beta$-cell dysfunction and death that need to be addressed in order to develop novel therapeutic approaches to block the detrimental effects of immune assault during diabetes progression and islet transplantation.

To improve our understanding of cytokine-induced gene expression changes, we chose to focus on myelin transcription factor 3 (Myt3), also known as suppressor of tumorigenicity 18 (St18), a C2HC-type zinc-finger transcription factor that is highly expressed in pancreatic islets. ${ }^{11}$ We previously established that exposure of islets to IL- $1 \beta$, TNF $a$ and IFN $\gamma$ suppresses Myt3 expression in a concentration- and timedependent manner. We further demonstrated that suppression of Myt3 in islets ex vivo, for as little as $48 \mathrm{~h}$, results in increased islet-cell death. ${ }^{12}$ These data suggested that Myt3 may participate in the development of diabetes downstream of immune assault. Here, to determine the role of Myt3 in islet function and survival in vivo we performed optimal and marginal mass syngeneic islet transplants and assessed glucose homeostasis and graft histology. We hypothesized that in this model Myt3 suppression would induce islet-cell apoptosis, supporting our hypothesis that Myt3 is a key regulator of islet-cell survival.

\section{Results}

Myt3 suppression impairs marginal but not optimal syngeneic islet graft function. To confirm the utility of syngeneic islet grafts as a model with which to study the in vivo effects of Myt3 suppression, we first rendered female C57/B6N mice diabetic via treatment with streptozotocin (STZ) and subsequently transplanted them with an optimal (300) mass of islets and assessed Myt3 expression in the

\footnotetext{
${ }^{1}$ Child and Family Research Institute, British Columbia Children's Hospital, 950 W28th Avenue, Vancouver, British Columbia, Canada V5Z 4H4; ${ }^{2}$ Department of Surgery, University of British Columbia, Vancouver, British Columbia, Canada V5Z 4E3 and ${ }^{3}$ Department of Pathology and Laboratory Medicine, University of British Columbia, Vancouver, British Columbia, Canada V6T 2B5

*Corresponding author: BG Hoffman, Diabetes Research Group, Child and Family Research Institute, British Columbia Children's Hospital, Room A4-185, 950 W28th Avenue, Vancouver, British Columbia, Canada V5Z 4H4. Tel: +1 6048752000 ext 4794; Fax: +1 604875 2373; E-mail: brad.hoffman@ubc.ca

Abbreviations: St18, suppression of tumorigenicity 18; Myt3, myelin transcription factor 3; INF $\gamma$, interferon-gamma; IL-1 $\beta$, interleukin-1 beta; TNF $\alpha$, tumour necrosis factor-alpha; qPCR, quantitative real-time polymerase chain reaction; MOI, multiplicity of infection; STZ, streptozotocin; IPGTT, intraperitoneal glucose tolerance tests; FACS, fluorescence-activated cell sorting; GFP, green fluorescence protein; TUNEL, terminal deoxynucleotidyl transferase dUTP nick end labelling; shRNA, short hairpin RNA; T1D, type 1 diabetes; T2D, type 2 diabetes; ECM, extracellular matrix

Received 08.1.16; revised 11.4.16; accepted 22.4.16; Edited by A Oberst
} 

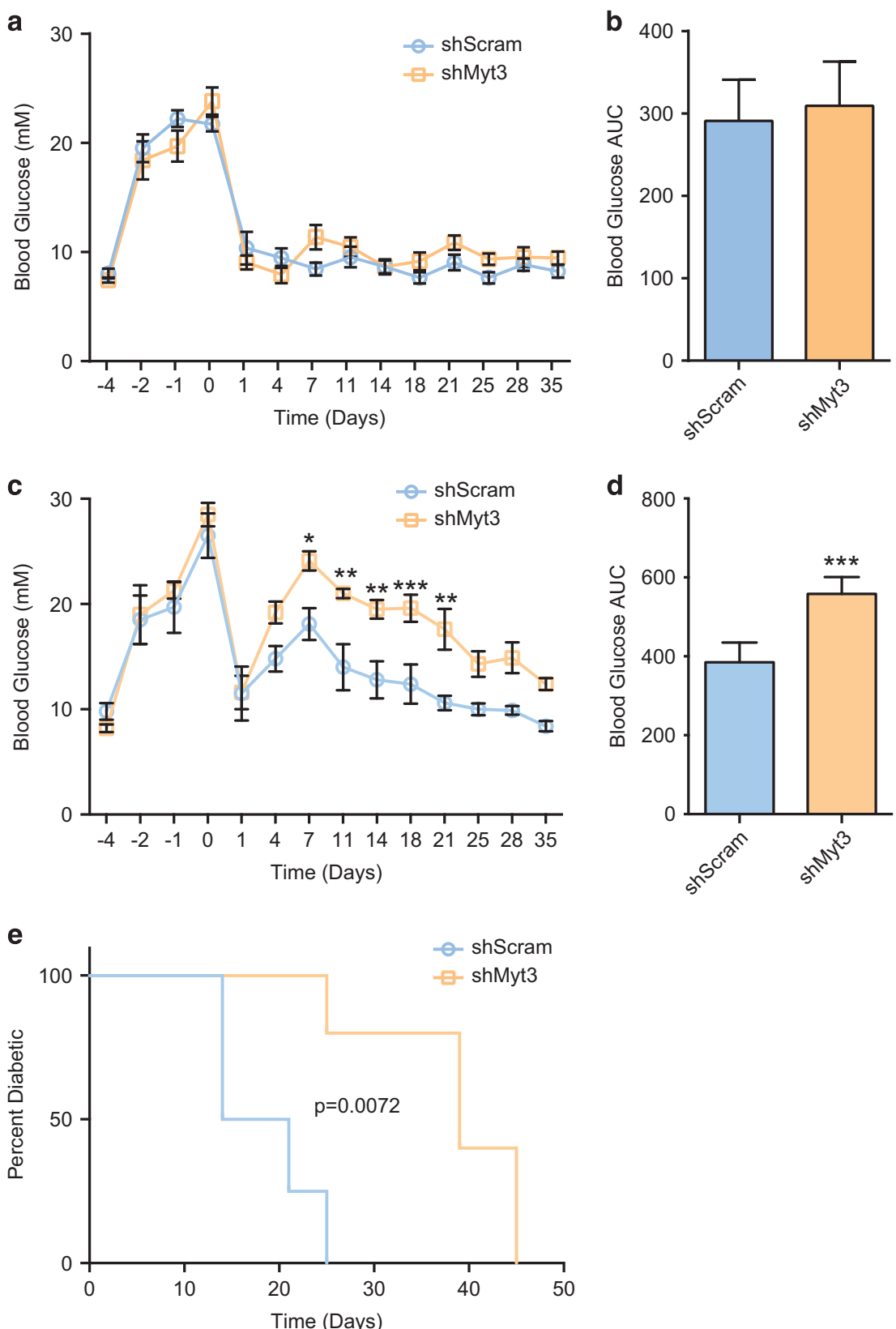

Figure 1 Myt3 suppression impairs marginal but not optimal islet graft function. (a) Random-fed blood glucose measurements for mice transplanted with an optimal mass (300 islets) of shScramble- or shMyt3-transduced islets. Results are represented as mean \pm S.E.M. of seven independent experiments. (b) Area under the curve for blood glucose measurements from day 1 to 35 post-optimal transplant. Results are represented as mean \pm S.E.M. of seven independent experiments. (c) Random-fed blood glucose measurements for mice transplanted with a marginal mass (150 islets) of shScramble- or shMyt3-transduced islets. Results are represented as mean \pm S.E.M. of five independent experiments. ${ }^{*} P<0.05,{ }^{* *} P<0.01,{ }^{* * *} P<0.001$ comparing mice receiving grafts using shMyt3-transduced islets with mice receiving grafts using shScrambletransduced islets (two-way ANOVA). (d) Area under the curve for blood glucose measurements from day 1 to 35 post-suboptimal transplant. Results are represented as mean \pm S.E.M. of five independent experiments. ${ }^{* * *} P<0.001$ comparing mice receiving grafts using shMyt3-transduced islets to mice receiving grafts using shScrambletransduced islets (unpaired t-test). (e) Kaplan-Meier survival curve comparing time to return to normoglycaemia for mice receiving marginal mass transplants of shScramble- or shMyt3-transduced islets. Mice were considered non-diabetic after two consecutive blood glucose measurements $<12 \mathrm{mM}$

grafts over 5 weeks. Myt3 expression during this time frame appeared to be maintained in the grafts at a similar level as in adult islets, indicating this is a suitable model for studying the effects of Myt3 suppression on graft survival and function (Supplementary Figure S1). As such, we transplanted STZdiabetic mice, as above, with either an optimal (300) or a marginal (150) mass of islets transduced with adenoviruses expressing an shRNA targeting Myt3 (shMyt3) or a scramble control (shScramble). Monitoring of random-fed blood glucose levels of mice receiving optimal grafts showed that both shScramble and shMyt3-transduced grafts were equally capable of normalizing blood glucose levels (Figures 1a 
and b). Further, there was no difference in the ability of shScramble or shMyt3 grafts to respond to a glucose challenge as determined by performing intraperitoneal glucose tolerance tests (IPGTT) 5 days or 5 weeks posttransplant (Supplementary Figures S2a-f).

On the other hand, mice transplanted with a marginal mass of shMyt3-transduced islets had significantly higher blood glucose levels from days 7 to 21 post-transplant $(P<0.05)$, as compared with mice transplanted with shScrambletransduced islets (Figures 1c and d). After day 21, the mice transplanted with shMyt3-transduced islets started to normalize, but still tended to have higher blood glucose levels, and had a significantly delayed time to reestablish normoglycaemia $(P<0.01$; two blood glucose measurements below $12 \mathrm{mM}$ ) (Figure 1e). At 5 weeks post-transplant, mice receiving shMyt3-transduced islets were also moderately less able to respond to a glucose challenge, but neither shScramble and shMyt3 grafts were able to re-stabilize blood glucose levels in the time frame of the IPGTT, and this difference was not significant (Supplementary Figures S2g-i). Taken together, these data suggest that, although in mice transplanted with an optimal mass of shMyt3-transduced islets sufficient $\beta$-cell mass remains to normalize blood levels, in marginal mass transplants Myt3 suppression impairs the ability of the grafts to establish normoglycaemia.

Myt3 suppression increases cell death in syngeneic islet transplants. To determine whether Myt3 suppression induced cell loss in the islet grafts, we performed immunohistochemistry on grafts harvested from optimal islet mass transplants, to focus on the direct effects of Myt3 on $\beta$-cell loss, in the absence of ER stress and other confounding factors that might occur in marginal mass transplants. FACS analysis of transduced islets prior to transplant, based on GFP (which is co-expressed with the shRNA), showed a transduction efficiency of 35-40\% for both shMyt3 and shScramble adenoviruses (Supplementary Figures S3a and b). Analysis of grafts 5 days post-transplant showed that shScrambletransduced grafts still contained approximately 40\% transduced cells, while shMyt3-transduced grafts contained only $\sim 25 \%$ transduced cells $(P<0.05)$ (Figures $2 a$ and $c)$. In contrast, culture of shScramble- or shMyt3-transduced islets in vitro for 5 days on the extracellular matrix 804G did not affect the number of GFP-positive islet cells (Supplementary Figures S3c and d), or significantly increase levels of apoptosis (Supplementary Figures S3e and f). Meanwhile, quantification of GFP area in the grafts 5 weeks post-transplant showed that shMyt3 grafts contained only 2-3\% GFP-positive cells, five times less than the number found in shScramble grafts $(13 \% ; P<0.001)$ (Figures $2 b$ and $c)$. Given that the cell loss seemed to be most rapid early in the engraftment period (nearly $40 \%$ of shMyt3-transduced cells were lost in the first 5 days after transplant), we assessed the number of cleaved caspase-3-positive cells 5 days post-transplant. At this time, shScramble-transduced grafts contained only a few cleaved caspase-3-positive cells $(\sim 1.7 \%)$, while shMyt3-transduced grafts contained three times as many cleaved caspase-3positive cells ( $5.3 \%)$ (Figures $2 \mathrm{~d}$ and e), indicating that Myt3 suppression significantly increased the level of apoptosis in the grafts at this time $(P<0.05)$.
The 35-40\% islet-cell transduction efficiency we achieved likely explains why no observable graft dysfunction was detectable in mice receiving an optimal mass of shMyt3transduced islets. Indeed, quantification of the number of $a$ - and $\beta$-cells in these grafts at both 5 days (Figures $3 a$ and $b$ ) and 5 weeks (Figures $3 c$ and d) post-transplant indicated that adequate numbers of $\alpha$ - and $\beta$-cells remain to maintain normoglycaemia despite the selective loss of shMyt3-transduced islet cells. Regardless, the fact that Myt3 suppression significantly increased the level of apoptosis in shMyt3transduced grafts after only 5 days, but did not significantly increase islet-cell apoptosis over the same time frame in vitro, suggests that Myt3 suppression sensitizes islet cells to undergo apoptosis in response to stresses faced specifically within the grafts.

Myt3 suppression increases chemokine expression but not immune infiltration. Cytokines produced by isletinfiltrating immune cells induce the expression of pro-inflammatory chemokines and cytokines in $\beta$-cells that act to recruit additional immune cells, contributing to $\beta$-cell dysfunction and apoptosis. ${ }^{2-4,6-8,13-18}$ Reanalysis of our previously generated RNA-seq data ${ }^{19}$ showed that Myt3 suppression induces the expression of several chemokines, including Ccl2, Cxcl10 and $\mathrm{Ccl} 20$ (Supplementary Figure S4a), and thus we sought to determine the significance of this increased chemokine expression to shMyt3-transduced islet-cell loss in our grafts. Quantitative real-time polymerase chain reaction (qPCR) validation demonstrated that a 2.4 -fold $(P<0.001)$ reduction in Myt3 expression resulted in a significant increase in the expression of $\mathrm{Ccl} 2$ (3.7-fold, $P \leqslant 0.05), C x c / 10$ (7.7-fold, $P \leqslant 0.05$ ) and Ccl20 (8.3-fold, $P \leqslant 0.05$ ) (Supplementary Figure S4b). To determine whether shMyt3-induced proinflammatory chemokine expression was sufficient to drive recruitment of immune cells, islet grafts were immunostained for the pan-immune cell marker CD45 at both 5 days and 5 weeks post-transplant. However, quantification of immune cell infiltration in the grafts showed that infiltration was negligible and equivalent between shScramble and shMyt3transduced islets (Supplementary Figure S4c). These data suggest that the level of chemokine production induced by Myt3 suppression is insufficient to drive additional recruitment of immune cells to syngeneic islet grafts.

Myt3 suppression sensitizes islet cells to metabolic stress-induced cell death. Given that Myt3 suppression induced a significant level of islet-cell loss during the time frame in which engraftment is occurring, we next sought to determine whether Myt3 suppression sensitized islet cells to undergo cell death in response to stresses experienced during this process. ${ }^{20,21}$ Specifically, during engraftment, islet transplants are exposed to significant metabolic stress, including hypoxia and nutrient deprivation prior to revascularization, hyperglycaemia upon initial revascularization, as well as oxidative stress, and stress induced by exposure to cytokines produced by immune cells recruited to the graft site. $^{21-25}$ We previously determined that cytokine exposure suppresses $M y t 3,{ }^{12}$ so we initially sought to determine the effect of these additional cell stresses on Myt3 expression. For this we exposed islet cells to cytokines $(0.4 \mathrm{ng} / \mathrm{ml} \mathrm{IFN} \gamma$, 
a

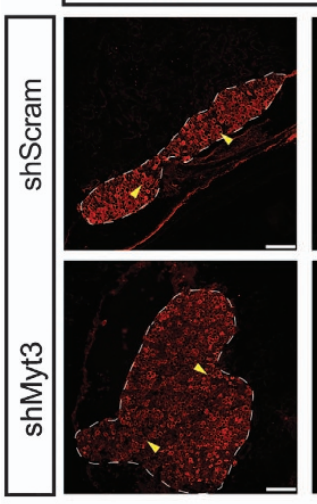

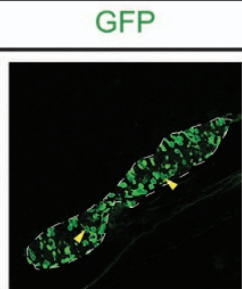
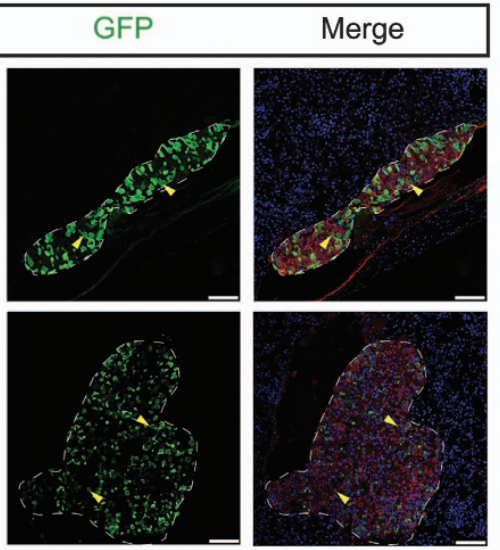

b
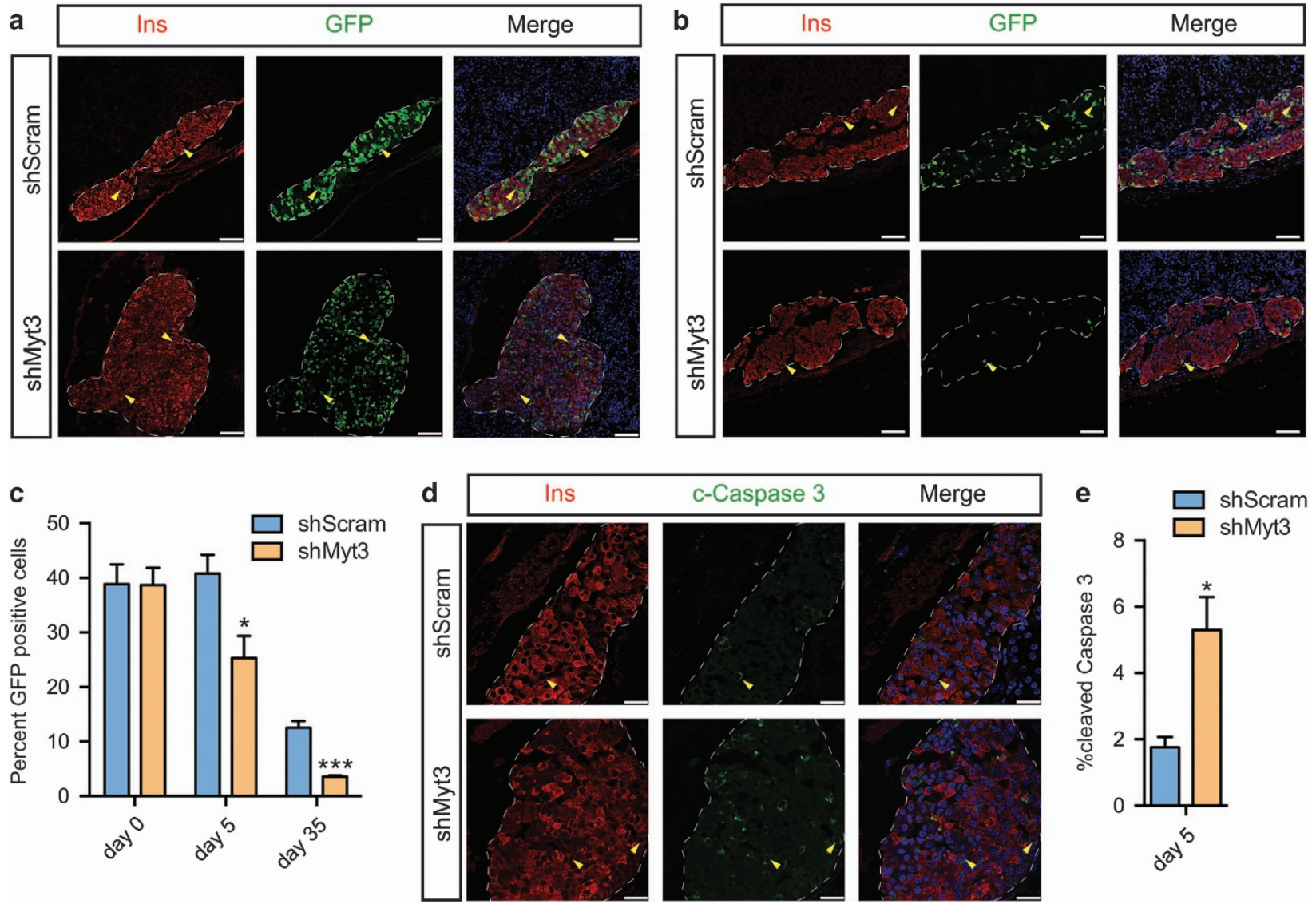

Figure 2 Loss of shMyt3-transduced cells occurs within 5 days of transplantation. shScramble- and shMyt3-transduced islet grafts (a) 5 days or (b) 5 weeks post-transplant were stained for insulin (red) and GFP (green) to mark transduced cells. Nuclei are labelled with Topro3 (blue). White dashed line represents the graft boundary. Yellow arrow heads indicate representative insulin and GFP co-positive cells. Scale bars: white bar $=75 \mu \mathrm{m}$. (c) Quantification of GFP area in shScramble-and shMyt3-transduced islet grafts pre-transplant, 5 days and 5 weeks post-transplant. Results are represented as mean \pm S.E.M. of three independent experiments. ${ }^{\star} P<0.05$, ${ }^{* \star *} P<0.001$ comparing grafts using shMyt3-transduced islets versus grafts using shScramble-transduced islets (unpaired $t$-test). (d) Grafts were stained with insulin (red) and cleaved caspase-3 (green). Nuclei are labelled with Topro3 (blue). White dashed line represents the graft boundary. Yellow arrow heads indicate representative insulin and cleaved caspase-3 co-positive cells. Scale bars: white bar $=25 \mu \mathrm{m}$. (e) Quantification of the percent of graft cells that stained for cleaved caspase-3. Results are represented as mean \pm S.E.M. of three independent experiments. ${ }^{*} P<0.05$ comparing grafts using shMyt3-transduced islets versus grafts using shScramble-transduced islets (unpaired $t$-test)

$0.07 \mathrm{ng} / \mathrm{ml} \mathrm{IL}-1 \beta$ and $0.04 \mathrm{ng} / \mathrm{ml}$ TNF $\alpha$ ), high glucose (30 mM glucose), low glucose $\left(2.8 \mathrm{mM}\right.$ glucose), hypoxia $\left(1 \% \mathrm{O}_{2}\right)$ or nutrient deprivation (serum withdrawal). This confirmed that Myt3 expression is inhibited by cytokine exposure (1.9-fold, $P<0.01)$ and demonstrated that hypoxia $(2.2$-fold, $P<0.01)$ and low glucose $(2.9$-fold, $P<0.01)$ similarly inhibit $M y t 3$ (Figure 4a). To determine whether Myt3 suppression sensitizes islet cells to these stresses, we transduced dispersed islet cells grown on 804G with our shMyt3 and shScramble adenoviruses and subsequently exposed the cells to each of the cell stressors and monitored cell death (by PI incorporation). Exposure of shMyt3-transduced islet cells to high glucose for $48 \mathrm{~h}$ increased the number of Pl-positive cells by 3.4-fold $(P<0.001)$ as compared with cells transduced with scramble control (Figure $4 \mathrm{~b})$, and 22.5 -fold $(P<0.001)$ as compared with shMyt3-transduced islets in normal glucose conditions. Myt3 suppression, however, did not significantly affect the level of cell death induced by any of the other stressors. These results indicate that Myt3 suppression sensitizes islet cells to high glucose-induced cell death.
Since Myt3 suppression sensitized islet cells specifically to high glucose-induced cell death, we next determined whether Myt3 suppression exacerbates hyperglycaemia-induced proapoptotic, ER- or oxidative-stress-related gene expression. Myt3 suppression resulted in a 1.7 -fold increase $(P<0.01)$ in high glucose-induced Bim expression as compared with shScramble-treated controls, but had little effect on the expression of other pro-apoptotic genes, or genes induced by ER- or oxidative stress (Figures $5 a-d$ ). Further, levels of $\mathrm{C} / \mathrm{EBP}$ homologous protein (CHOP), or phosphorylated eukaryotic initiation factor-2 (elF2a), were also unaltered, implying Myt3 suppression does not affect hyperglycaemiainduced ER stress in this context (Figures $5 \mathrm{e}-\mathrm{g}$ ).

These results suggest that Myt3 suppression may specifically sensitize islet cells to high glucose-induced cell death via Bim. In support of this, Myt3 suppression resulted in an approximately fivefold increase $(P<0.001)$ in Bim protein levels (Figures $6 a$ and $b$ ) in cells treated with high glucose. Next, we measured islet-cell death in shMyt3-transduced cells exposed to high glucose and co-transduced with a lentivirus 

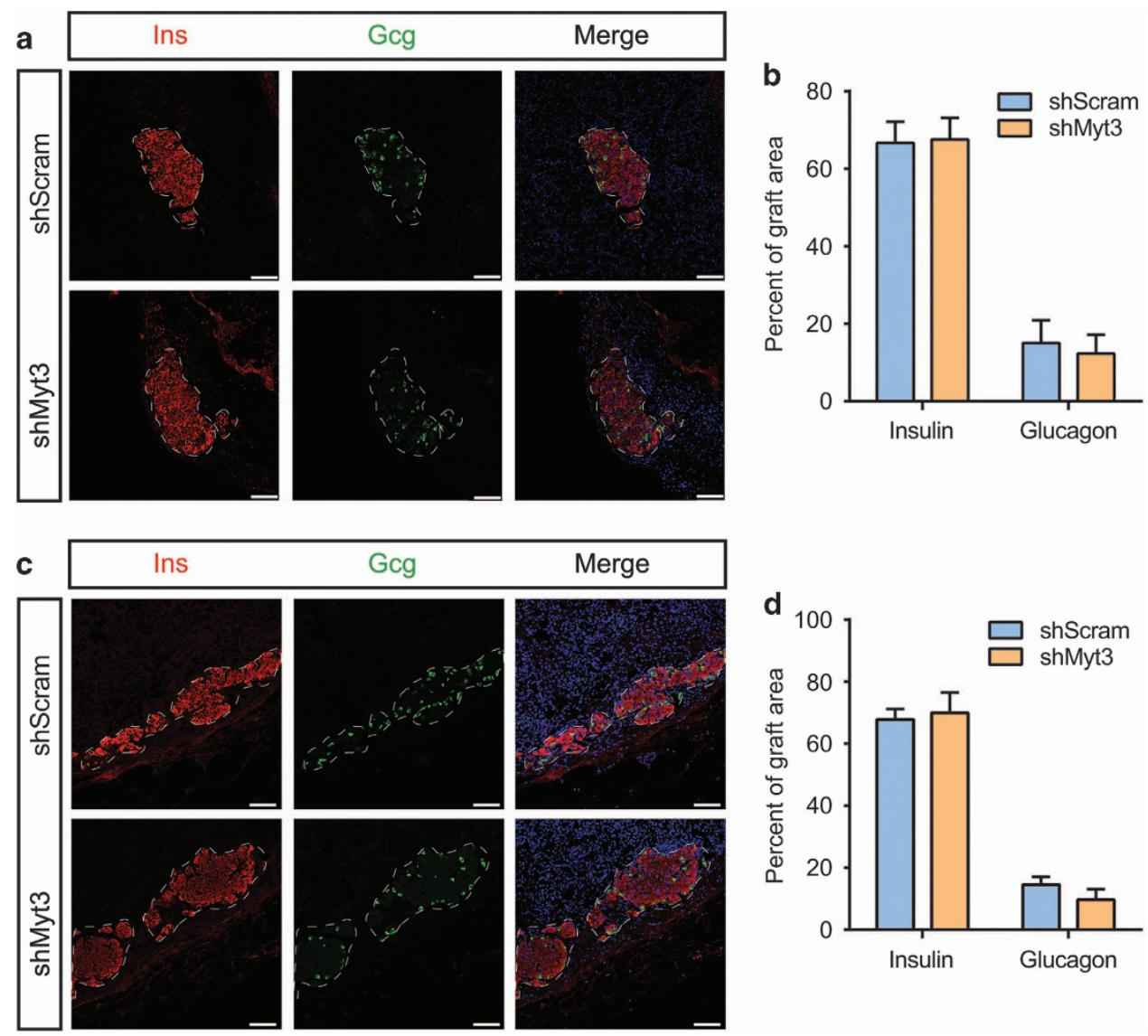

Figure 3 shMyt3-transduced cells are lost from grafts without altering graft composition. (a) shScramble-and shMyt3-transduced islet grafts 5 days post-transplant were stained for insulin (red) and glucagon (green) to mark transduced cells. Nuclei are labelled with Topro3 (blue). White dashed lines represent the graft boundaries. Scale bars: white bar $=75 \mu \mathrm{m}$. (b) Quantification of insulin and glucagon area in (a). Results are represented as mean \pm S.E.M. of three independent experiments. (c) shScramble-and shMyt3transduced islet grafts 5 weeks post-transplant were stained with insulin (red) and glucagon (green). Nuclei are labelled with Topro3 (blue). White dashed lines represent the graft boundaries. Scale bars: white bar $=75 \mu \mathrm{m}$. (d) Quantification of insulin and glucagon area in (c). Results are represented as mean \pm S.E.M. of three independent experiments
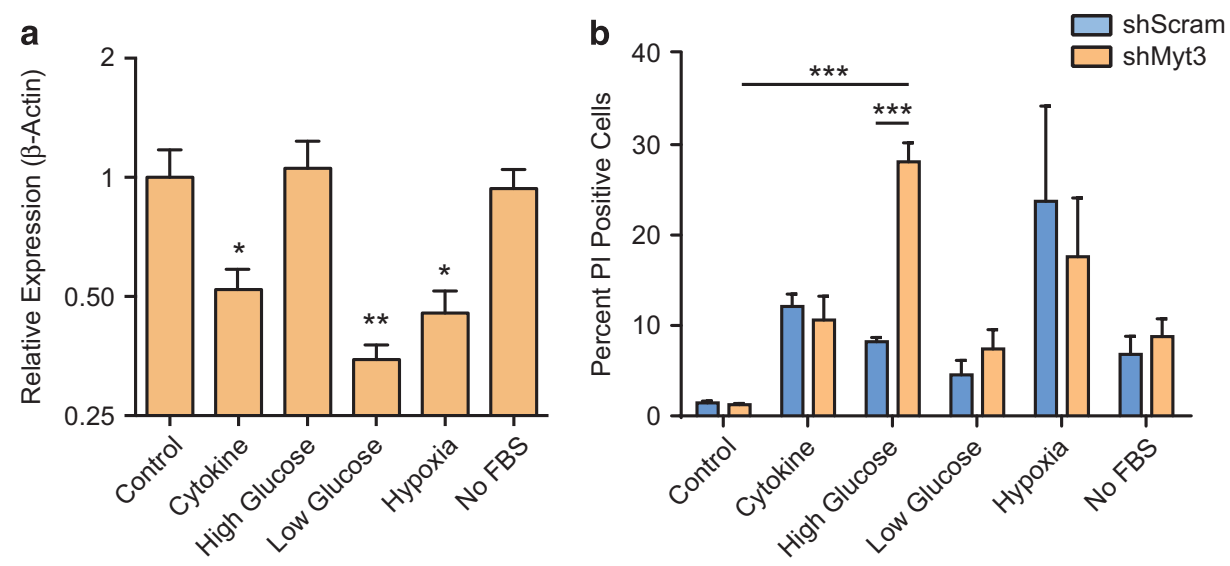

Figure 4 Myt3 suppression sensitizes islet cells to high glucose-induced cell death. (a) Myt3 expression was determined by qPCR following exposure to either $11 \mathrm{mM}$ glucose (Control), $0.4 \mathrm{ng} / \mathrm{ml} \mathrm{IFN} \gamma, 0.07 \mathrm{ng} / \mathrm{ml} \mathrm{IL-1} \beta$ and $0.04 \mathrm{ng} / \mathrm{ml} \mathrm{TNF} \alpha$ (Cytokine), high glucose ( $30 \mathrm{mM}$ glucose), low glucose ( $2.8 \mathrm{mM}$ glucose), hypoxia ( $1 \% \mathrm{O}_{2}$ ) or serum starvation (No FBS). Results are represented as mean \pm S.E.M. of four independent experiments. ${ }^{*} P<0.05,{ }^{* *} P<0.01$ comparing islet cells exposed to the indicated treatments as compared with $11 \mathrm{mM}$ glucose (Control) (one-way ANOVA). (b) Cell death was measured by propidium iodide incorporation in dispersed islet cells transduced with shScramble or shMyt3 adenoviruses were exposed to either $11 \mathrm{mM}$ glucose (Control), $0.4 \mathrm{ng} / \mathrm{ml} \mathrm{IFN} \gamma, 0.07 \mathrm{ng} / \mathrm{ml} \mathrm{IL-1 \beta}$ and $0.04 \mathrm{ng} / \mathrm{ml}$ TNF $\alpha$ (Cytokine), high glucose ( $30 \mathrm{mM}$ glucose), low glucose $\left(2.8 \mathrm{mM}\right.$ glucose), hypoxia $\left(1 \% \mathrm{O}_{2}\right)$ or serum starvation (No FBS). Results are represented as mean \pm S.E.M. of five independent experiments. ${ }^{* \star *} P<0.001$ comparing the indicated bars (two-way ANOVA) 

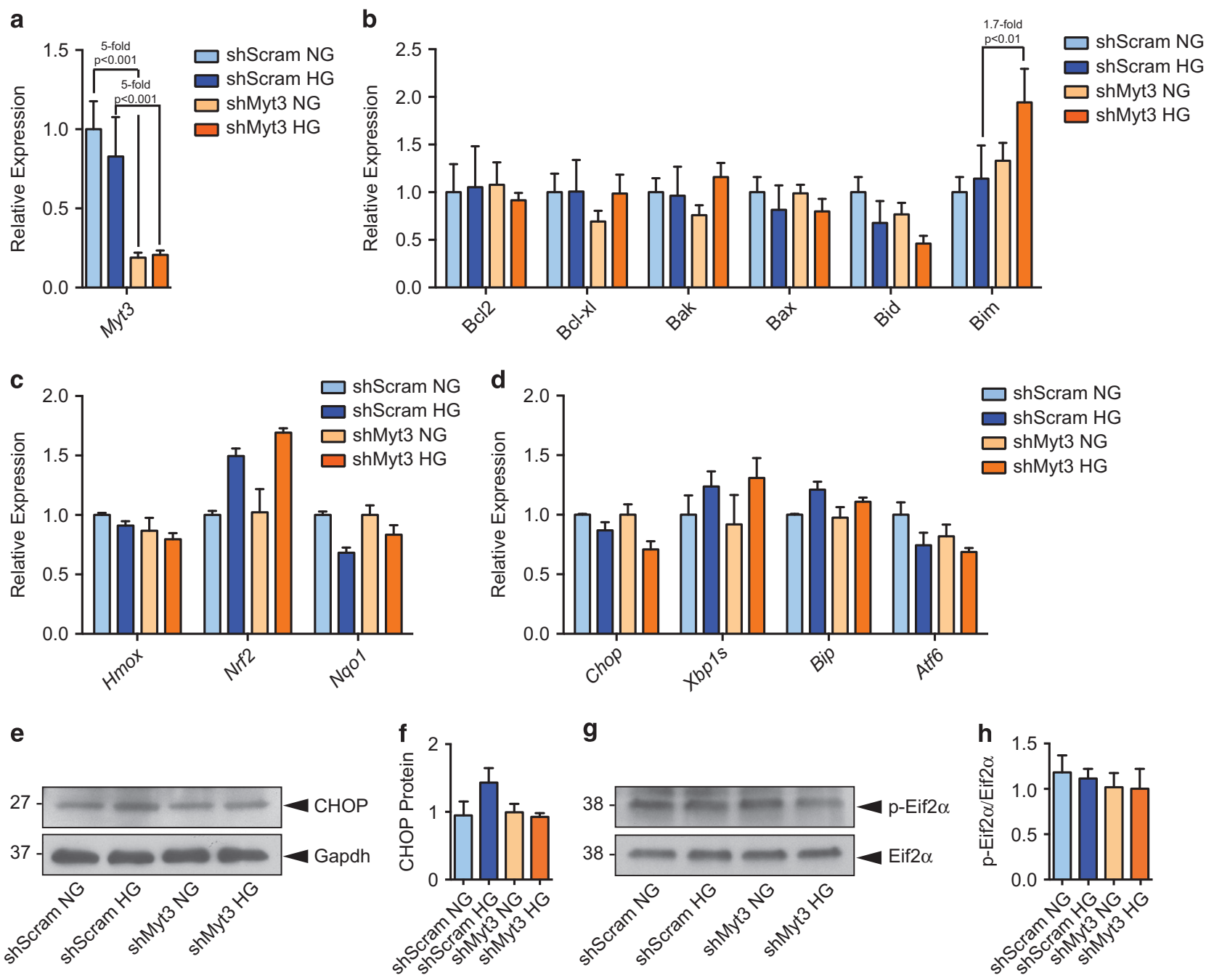

Figure 5 Myt3 suppression increases high glucose-induced Bim expression in islet cells. qPCR analysis of (a) Myt3 expression, (b) pro-and anti-apoptotic Bcl-family genes and (c) ER stress or (d) oxidative stress-induced genes, in shScramble- or shMyt3-transduced islets under normal glucose (NG) or high glucose (HG) conditions. (e) CHOP and (g) phosphorylated elF2 $\alpha$ versus total elF2 $\alpha$ protein levels were determined by western blot in shScramble- or shMyt3-transduced islets under NG or HG conditions. (f) Quantification of CHOP protein as a fraction of Gapdh protein in (c). Results are represented as mean \pm S.E.M. of three independent experiments. (h) Quantification of phosphorylated elF2 $\alpha$ as a fraction of total elF2 $\alpha$ protein levels in $(\mathbf{g})$. Results are represented as mean \pm S.E.M. of three independent experiments

expressing an shRNA targeting Bim, which resulted in a five-fold reduction in Bim expression (Figure 6c). Bim suppression essentially abrogated the increase in shMyt3transduced islet-cell apoptosis in response to high glucose, as compared with shScramble-transduced controls (Figure 6d). These data indicate that exposure of islet cells, in which Myt3 is suppressed, to high glucose results in Bim upregulation, causing an imbalance in pro-apoptotic and pro-survival Bcl2 family members that results in islet-cell death.

\section{Discussion}

During diabetes progression exposure of $\beta$-cells to cytokines produced by infiltrating immune cells activates pro-inflammatory signalling cascades that ultimately lead to amplification of inflammatory signals and initiation of apoptosis. ${ }^{2-4,6-8,13-17}$ We previously demonstrated that the transcription factor Myt3 is one of the downstream targets of IL-1 $1 \beta$, TNF $\alpha$ and IFN $\gamma$ signalling in $\beta$-cells, and that its suppression is sufficient to induce a two-fold increase in apoptosis when islet cells are cultured in vitro, without affecting islet function. ${ }^{12}$ However, these analyses were conducted over a very short period $(\sim 48 \mathrm{~h})$, in the absence of extracellular matrix, and did not provide insight into the longer-term effects of Myt3 suppression on islet function and survival in vivo. In the current work, we sought to address this deficit using a syngeneic islet transplant model to examine the effects of Myt3 suppression on graft survival and function.

We demonstrate that, although neither body weight nor blood glucose homoeostasis were significantly affected in mice transplanted with an optimal mass of shMyt3-transduced islets, in marginal mass transplants Myt3 suppression substantially delays the time required for graft recipients to reestablish normoglycaemia. Further, in optimal mass transplants, by 5 weeks post-transplant only $2-3 \%$ of cells remaining in shMyt3-transduced islet grafts were shMyt3-transduced 
a

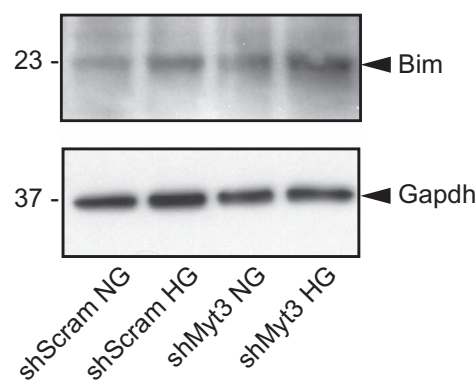

b
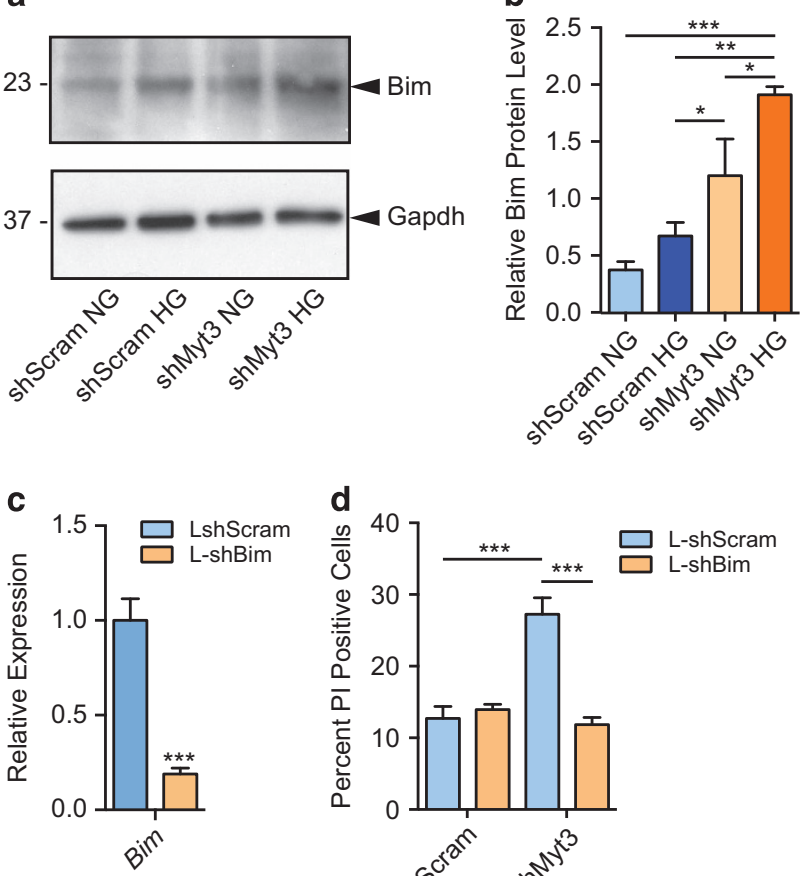

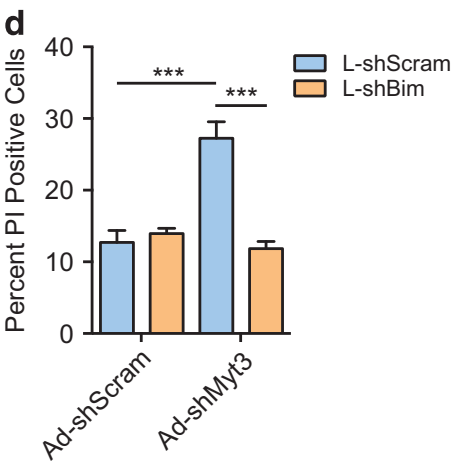

Figure 6 Myt3 suppression sensitizes islet cells to high glucose-induced cell death via upregulation of Bim. (a) Bim protein was determined by western blot in shScramble- or shMyt3-transduced islets under normal glucose (NG) or high glucose (HG) conditions. (b) Quantification of Bim protein as a fraction of Gapdh protein in (a). Results are represented as mean \pm S.E.M. of three independent experiments. ${ }^{\star} P<0.05,{ }^{* *} P<0.01,{ }^{* * *} P<0.001$ comparing the indicated bars (two-way ANOVA). (c) qPCR analysis of Bim expression in dispersed islet cells transduced with shScramble (L-shScram) or shBim (L-shBim) expressing lentiviruses. Results are represented as mean \pm S.E.M. of four independent experiments. ${ }^{* * *} P<0.001$ comparing L-shScram to L-shBim transduced cells (unpaired $t$-test). (d) Cell death was determined by propidium iodide incorporation in dispersed islet cells cotransduced with lentiviral shScramble (L-shScram) or shBim (L-shBim), and adenoviral shScramble (Ad-shScram) or shMyt3 (Ad-shMyt3), under high glucose conditions. Results are represented as mean \pm S.E.M. of four independent experiments. ${ }^{* * *} P<0.001$ comparing the indicated bars (two-way ANOVA)

cells. Although, there was also some loss of GFP expression in shScramble-transduced islet grafts at this time, given that our initial transduction efficiency was $\sim 40 \%$, and the presence of five times fewer GFP-positive cells in the shMyt3 grafts than in the shScramble grafts, this low frequency of shMyt3-transduced cells in the grafts at this time suggests that the majority of shMyt3-transduced cells had undergone cell death. In support of this, Myt3 suppression significantly increased the level of apoptosis in optimal mass grafts after only 5 days, and the loss of transduced cells was already evident at this time. Importantly, suppression of Myt3 did not have any significant effect on islet-cell death in islet cells grown in vitro on the extracellular matrix 804G for the same amount of time. These data suggest that Myt3 suppression sensitizes islet cells to undergo apoptosis in response to stresses faced within the islet graft.

Myt3 suppression in islets in vitro leads to the upregulation of several chemokines, likely via Myt3 suppression indirectly or directly allowing their de-repression, given that islet-cell death was not significantly increased by Myt3 suppression in these conditions. As the chemokines induced by Myt3 suppression are capable of recruiting immune cells, we hypothesized their expression might contribute to the loss of islet cells following transplantation. ${ }^{2-4,6-8,13-17}$ However, the level of induction of these chemokines appeared to be insufficient to drive the recruitment of additional immune cells to the grafts, suggesting alternate mechanisms are likely responsible for the increased cell death observed in the shMyt3-transduced islet grafts.

Islet transplants are exposed to significant levels of cell stress during the engraftment process. ${ }^{21}$ The increased cell death observed during the first 5 days post-transplant in our model implicated these cell stresses as a potential modifier of shMyt3-induced islet-cell death. In fact, analysis of various cell stressors demonstrated that Myt3 suppression significantly increased high glucose-induced islet-cell death. In the context of transplantation, this suggests that loss of Myt3 promotes cell death when the engrafted islets are exposed to high circulating concentrations of blood glucose immediately following transplantation. Myt3 suppression did not further sensitize islet cells to cytokine-, low glucose- or hypoxiainduced cell death, likely because these stressors already reduce Myt3 expression. We further show that the sensitivity of islet cells to high glucose-induced cell death following Myt3 suppression is dependent on the induction of the pro-apoptotic Bcl2 family member Bim. Bim induction is similarly critical to islet-cell death in response to Pdx1 and IRS2 deficiency and in response to glucotoxity. ${ }^{26-28}$

Exposure of $\beta$-cells to inflammatory cytokines can exacerbate the effects of glucotoxicity; however, how this 'glucocytotoxicity' occurs is not well characterized. ${ }^{29-31}$ Our previous data show that suppression of Myt3 by inflammatory cytokines plays a significant role in cytokine-induced $\beta$-cell apoptosis, while our data here indicate that Myt3 suppression sensitizes $\beta$-cells to high glucose-induced cell death. Together, these data suggest that Myt3 may act as a mediator of inflammatory and glucotoxic signalling pathway crosstalk, with inflammatory signalling-induced Myt3 suppression, as may occur in T1D and T2D, sensitizing $\beta$-cells to undergo cell death in response to hyperglycaemic episodes via allowing precocious Bim activation. We believe that future efforts need to be targeted at uncovering the mechanisms underlying the effects of Myt3 suppression on $\beta$-cell apoptosis and determining whether the maintenance of Myt3 levels can reduce $\beta$-cell death and dysfunction.

\section{Materials and Methods}

Mouse maintenance and handling. Mice were maintained according to the guidelines of the Canadian Council on Animal Care using protocols approved by the UBC Animal Care Committee. Mice were housed in a group setting of up to five mice per cage with daily health checks and cage maintenance performed by animal facility staff members. In our colony, mice were maintained in a 12-h light/dark cycle, fed a standard low fat diet (LabDiet, St. Louis, MO, USA) ad libitum and their environment was enriched through administration of sunflower seeds and hiding places. Mice were housed in this setting until needed for experiments. C57/B6N (812 weeks old) mice were used in all studies.

Mice used in non-survival procedures were anaesthetized using isofluorane inhalation followed by cervical dislocation. Toe pinch reflex was checked prior to cervical dislocation in order to ensure that the animals were at a surgical plane of anaesthesia 
Islet isolation and culture. Pancreata from 8- to 12-week-old female mice were perfused with $1000 \mathrm{U} / \mathrm{ml}$ Collagenase XI (Sigma-Aldrich, Oakville, ON, Canada) and incubated for $15 \mathrm{~min}$ at $37^{\circ} \mathrm{C}$. Pancreata were manually disrupted and passed through a $70 \mu \mathrm{M}$ filter (Corning, New York, NY, USA) and islets were handpicked. Islets were cultured in RPMI 1640 (11 mM glucose) supplemented with $10 \%$ FBS, 50U/ml penicillin/streptomycin and $2 \mathrm{mM}$ L-glutamine at $37^{\circ}$ in a $5 \% \mathrm{CO}_{2}$ humidified incubator. All tissue culture reagents were purchased from ThermoFisher (Burlington, ON, Canada). Islets were transduced with the shScramble or shMyt3 adenoviruses as done previously ${ }^{12}$ and cultured for $48 \mathrm{~h}$ prior to transplantation.

Islet transplantation. Transplant recipient mice were rendered diabetic by injection of $230 \mathrm{mg} / \mathrm{kg}$ streptozotocin (Sigma-Aldrich) into the intraperitoneal space. We monitored these animals on a daily basis for signs of illness and measured blood glucose using a One Touch Ultra glucose test meter (LifeScan Canada Ltd, Burnaby, BC, Canada) to determine the induction of hyperglycaemia. Following development of streptozotocin-induced diabetes in these mice, they were transplanted under the kidney capsule with 300 islets for optimal mass transplants or 150 islets for marginal mass transplants. One hour before surgery, mice received subcutaneous injection of a combination of meloxicam $(1 \mathrm{mg} / \mathrm{kg})$ and buprenorphine $(0.1 \mathrm{mg} / \mathrm{kg})$. The mice were anaesthetized for surgery with isofluorane $(2 \%$ in $2 \mathrm{l} / \mathrm{min}$ oxygen flow), and surgical plane of anasthesia was assessed by toe pinch reflex. A small nick was made in the kidney capsule and the islets were slowly injected below the capsule. The incisions were sutured closed and the mice were allowed to recover from the anaesthetic prior to being returned to their cages. ${ }^{32}$

Glucose tolerance tests. In vivo islet function was assessed by intraperitoneal glucose tolerance test (IPGTT). Mice were fasted for $6 \mathrm{~h}$ and injected with $1 \mathrm{~g} / \mathrm{kg}$ D50 glucose (Sigma-Aldrich), and saphenous blood glucose was measured at $0,15,30,60$ and $120 \mathrm{~min}$.

Immunohistochemistry. Immunohistochemistry was performed on paraffin sections of islets embedded under the kidney capsule. Sections were stained with goat anti-glucagon (1:500; Santa Cruz, Dallas, TX, USA), guinea pig anti-insulin (1:500; Linco, St. Charles, MO, USA), rabbit anti-GFP (1:1000; MBL, Woburn, MA, USA) and rabbit anti-cleaved caspase-3 (1:500; Santa Cruz). Primary antibodies were detected using donkey anti-rabbit Alexa 488 (1:1000; Molecular Probes, Eugene, OR, USA), goat anti-guinea pig Alexa 546 (1:500; Molecular Probes) or donkey anti-goat Alexa 488 (1/1000; Molecular Probes).

Cell death assay. Islets were isolated as described above and a single-cell suspension was prepared by trypsinization. Two hundred islet equivalents per well were plated in 24-well tissue culture plates treated with 804G, a complete extracellular matrix (ECM) produced by a rat bladder carcinoma cell line. ${ }^{33}$ Cells were transduced with the shScramble or shMyt3 adenoviruses overnight at an $\mathrm{MOI}$ of 10 and cultured in the presence or absence of various cell stressors for a further $48 \mathrm{~h}$. RPMl media supplemented with $11 \mathrm{mM}$ glucose (normal glucose) served as the control condition, while stressors included a cytokine cocktail $(0.4 \mathrm{ng} / \mathrm{ml} \mathrm{IFN} \gamma, 0.07 \mathrm{ng} / \mathrm{ml} \mathrm{IL-1} \beta$ and $0.04 \mathrm{ng} / \mathrm{ml} \mathrm{TNF} \alpha$ ), serum starvation, low glucose ( $2.8 \mathrm{mM}$ glucose), high glucose (30 mM glucose) or hypoxia $\left(1 \% \mathrm{O}_{2}\right)$. The pLKO-Bim shRNA (TRCN00000096929695), which targets all Bim isoforms, ${ }^{26}$ and lentiviral control (RHS6848) vectors were purchased from GE Dharmacon (Ottawa, ON, Canada). shScramble and shMyt3transduced islets were co-transduced with $2.5 \mu$ l lentiviral shScramble or $\operatorname{shBim}\left(1 \times 10^{9}\right.$ pfu) overnight. The media was changed to fresh $30 \mathrm{mM}$ glucose RPMl and cells were cultured for a further $48 \mathrm{~h}$. We added $1 \mu \mathrm{l}$ propidium iodide (PI, $1 \mathrm{mg} / \mathrm{ml}$; Sigma-Aldrich) and $0.5 \mu \mathrm{l}$ Hoescht to each well $24 \mathrm{~h}$ prior to imaging. Cells were imaged with an SP8 confocal microscope (Leica, Wetzlar, Germany) and quantification was performed with CellProfiler image analysis software (Broad Institute, Cambridge, MA, USA).

qPCR. Dispersed islets were either transduced as above or left untransduced and cultured in RPMI media supplemented with the cell stressors described above for $36 \mathrm{~h}$. RNA was isolated using Trizol and the PureLink RNA purification kit (Ambion, Burlington, ON, Canada). cDNA was generated using Superscript III (Invitrogen, Burlington, ON, Canada). Taqman probes were used to quantify $\beta$-actin and Myt3, all other primers were designed using Primer3plus and ordered from IDT. A Viia7 real-time PCR system and SYBR Green supermix (Applied Biosystems, Burlington, ON, Canada) or Taqman Fast Advanced Master Mix (Applied Biosystems) was used for all reactions. cDNA (10ng) was used in each reaction, with all reactions done in triplicate. $\beta$-Actin was used as an internal control and the change in expression was calculated using $2^{-\Delta \Delta C t}$.
Western blot analysis. Cell lysates were prepared from transduced, dispersed islets cultured under normal glucose (11 mM glucose) or high glucose ( $30 \mathrm{mM}$ glucose) conditions for $48 \mathrm{~h}$. Cells were recovered by trypsinization and lysed by sonication in RIPA buffer (ThermoFisher). Twenty-five micrograms total protein was loaded in each well of a 4-15\% TGX gel (Biorad, Mississauga, ON, Canada). Gels were transferred to $0.2 \mu \mathrm{m}$ polyvinyl difluoride membranes (Biorad) using the Trans-Blot Turbo apparatus (Biorad) according to the manufacturer's instructions. Membranes were probed with antibodies against Bim (1:500; Cell Signaling, Danvers, MA, USA), CHOP (1:500; Santa Cruz), phospho-elF2 $\alpha$ (1:1000; Cell Signaling) and total elF2 $\alpha$ (1:500; Abcam, Cambridge, MA, USA) at $4{ }^{\circ} \mathrm{C}$ in $5 \%$ skim milk diluted in TBS supplemented with $0.05 \%$ Tween-20. Blots were subsequently stripped using the Abcam mild stripping protocol and re-probed with anti-Gapdh (1:10000; Cell Signaling). Donkey anti-Rabbit or anti-Mouse (Santa Cruz) HRP-conjugated secondary antibodies were used at 1:10 000 in PBS for $1 \mathrm{~h}$ at room temperature. Membranes were treated with ECL reagent (ThermoFisher) for $1 \mathrm{~min}$ prior to developing films.

Statistical analysis. Statistical analysis was performed using a two-way ANOVA with a Sidak's multiple comparison test for physiological measurements over time. Statistical analysis was performed using an ANOVA with a Tukey's multiple comparison test for qPCR, western blot and cell death measurements. All other data were analysed using an unpaired, two-tailed Student's $t$-test. Data from at least three independent experiments are represented as mean \pm S.E.M. Statistical significance was accepted at $P$-values $<0.05$.

\section{Conflict of Interest}

The authors declare no conflict of interest.

Acknowledgements. We acknowledge Galina Soukatcheva for performing islet transplants. We also thank Dr. Dan Luciani for allowing us the use of his confocal microscope. Additionally, we would like to thank the staff of the Animal Care Facility at the CFRI for daily maintenance of the mouse colonies. Funding was provided by the Child and Family Research Institute, the Canadian Institutes for Health Research (MOP-111010 to BGH), the JDRF (1-2011-550 to CBV) and the Canadian Diabetes Association (OG-3-11-3413-CV to CBV). BRT and BGH are supported by fellowships from the Canadian Diabetes Association.

1. Atkinson MA. The pathogenesis and natural history of type 1 diabetes. Cold Spring Harb Perspect Med 2012; 2: a007641.

2. Delovitch TL, Singh B. The nonobese diabetic mouse as a model of autoimmune diabetes: immune dysregulation gets the NOD. Immunity 1997; 7: 727-738.

3. Kay TW, Thomas HE, Harrison LC, Allison J. The beta cell in autoimmune diabetes: many mechanisms and pathways of loss. Trends Endocrinol Metab 2000; 11: 11-15.

4. Eizirik DL, Colli ML, Ortis F. The role of inflammation in insulitis and beta-cell loss in type 1 diabetes. Nat Rev Endocrinol 2009; 5: 219-226.

5. Eizirik DL, Sandler S, Welsh N, Cetkovic-Cvrlje M, Nieman A, Geller DA et al. Cytokines suppress human islet function irrespective of their effects on nitric oxide generation. $J$ Clin Invest 1994; 93: 1968-1974.

6. Ortis F, Pirot P, Naamane N, Kreins AY, Rasschaert J, Moore F et al. Induction of nuclear factor-kappaB and its downstream genes by TNF-alpha and IL-1beta has a pro-apoptotic role in pancreatic beta cells. Diabetologia 2008; 51: 1213-1225.

7. Suk K, Kim S, Kim YH, Kim K-A, Chang I, Yagita H et al. IFN-gamma/TNF-alpha synergism as the final effector in autoimmune diabetes: a key role for STAT1/IFN regulatory factor-1 pathway in pancreatic beta cell death. $J$ Immunol 2001; 166: 4481-4489.

8. Cardozo AK, Heimberg H, Heremans $Y$, Leeman R, Kutlu B, Kruheffer $M$ et al. A comprehensive analysis of cytokine-induced and nuclear factor-kappa B-dependent genes in primary rat pancreatic beta-cells. J Biol Chem 2001; 276: 48879-48886.

9. Kutlu B, Cardozo AK, Darville MI, Kruhøffer M, Magnusson N, Ørntoft T et al. Discovery of gene networks regulating cytokine-induced dysfunction and apoptosis in insulin-producing INS-1 cells. Diabetes 2003; 52: 2701-2719.

10. Sarkar SA, Kutlu B, Velmurugan K, Kizaka-Kondoh S, Lee CE, Wong R et al. Cytokinemediated induction of anti-apoptotic genes that are linked to nuclear factor kappa-B (NF-kappaB) signalling in human islets and in a mouse beta cell line. Diabetologia 2009; 52: 1092-1101.

11. Hoffman BG, Zavaglia B, Witzsche J, Ruiz de Algara T, Beach M, Hoodless PA et al. Identification of transcripts with enriched expression in the developing and adult pancreas. Genome Biol 2008; 9: R99.

12. Tennant BR, Islam R, Kramer MM, Merkulova Y, Kiang RL, Whiting CJ et al. The transcription factor Myt3 acts as a pro-survival factor in $\beta$-cells. PLoS One 2012; 7: e51501. 
13. van Belle TL, Coppieters KT, von Herrath MG. Type 1 diabetes: etiology, immunology, and therapeutic strategies. Physiol Rev 2011; 91: 79-118.

14. Kim S, Kim HS, Chung KW, Oh SH, Yun JW, Im S-H et al. Essential role for signal transducer and activator of transcription-1 in pancreatic beta-cell death and autoimmune type 1 diabetes of nonobese diabetic mice. Diabetes 2007; 56: 2561-2568.

15. Cnop M, Welsh N, Jonas J-C, Jörns A, Lenzen S, Eizirik DL. Mechanisms of pancreatic betacell death in type 1 and type 2 diabetes: many differences, few similarities. Diabetes 2005; 54 (Suppl 2): S97-S107.

16. Johnson JD, Luciani DS. Mechanisms of pancreatic beta-cell apoptosis in diabetes and its therapies. Adv Exp Med Biol 2010; 654: 447-462.

17. Eizirik DL, Mandrup-Poulsen T. A choice of death-the signal-transduction of immunemediated beta-cell apoptosis. Diabetologia 2001; 44: 2115-2133.

18. Cardozo AK, Kruhøffer M, Leeman R, Ørntoft T, Eizirik DL. Identification of novel cytokineinduced genes in pancreatic beta-cells by high-density oligonucleotide arrays. Diabetes 2001; 50: 909-920.

19. Tennant BR, Chen J, Shih AZL, Luciani DS, Hoffman BG. Myt3 mediates Laminin-V/Integrin$\beta 1$-induced islet cell migration via Tgfbi. Mol Endocrinol 2015; 29: 1254-1268.

20. Lau J, Carlsson P-O. Low revascularization of human islets when experimentally transplanted into the liver. Transplantation 2009; 87: 322-325.

21. Carlsson P-O. Influence of microenvironment on engraftment of transplanted $\beta$-cells. Ups J Med Sci 2011; 116: 1-7.

22. Zheng X, Zheng X, Wang X, Ma Z, Gupta Sunkari V, Botusan I et al. Acute hypoxia induces apoptosis of pancreatic $\beta$-cell by activation of the unfolded protein response and upregulation of CHOP. Cell Death Dis 2012; 3: e322.

23. Bennet W, Groth CG, Larsson R, Nilsson B, Korsgren O. Isolated human islets trigger an instant blood mediated inflammatory reaction: implications for intraportal islet transplantation as a treatment for patients with type 1 diabetes. Ups J Med Sci 2000; 105: 125-133.

24. Carlsson P-O, Palm F, Mattsson G. Low revascularization of experimentally transplanted human pancreatic islets. J Clin Endocrinol Metab 2002; 87: 5418-5423.

25. Yasunami Y, Kojo S, Kitamura H, Toyofuku A, Satoh M, Nakano M et al. Valpha14 NK $T$ cell-triggered IFN-gamma production by Gr-1+CD11b+ cells mediates early graft loss of syngeneic transplanted islets. J Exp Med 2005; 202: 913-918.

26. Ren D, Sun J, Wang C, Ye H, Mao L, Cheng EH et al. Role of BH3-only molecules Bim and Puma in $\beta$-cell death in Pdx1 deficiency. Diabetes 2014; 63: 2744-2750.
27. Ren D, Sun J, Mao L, Ye H, Polonsky KS. BH3-only molecule Bim mediates $\beta$-cell death in IRS2 deficiency. Diabetes 2014; 63: 3378-3387.

28. Wali JA, Rondas D, McKenzie MD, Zhao Y, Elkerbout L, Fynch S et al. The proapoptotic BH3-only proteins Bim and Puma are downstream of endoplasmic reticulum and mitochondrial oxidative stress in pancreatic islets in response to glucotoxicity. Cell Death Dis 2014; 5: e1124.

29. Sandler S, Bendtzen K, Eizirik DL, Strandell E, Welsh M, Welsh N. Metabolism and beta-cell function of rat pancreatic islets exposed to human interleukin- 1 beta in the presence of a high glucose concentration. Immunol Lett 1990; 26: 245-251.

30. Dunger A, Schröder D, Augstein P, Witstruck T, Wachlin G, Vogt L et al. Impact of metabolic activity of beta cells on cytokine-induced damage and recovery of rat pancreatic islets. Acta Diabetol 1995; 32: 217-224.

31. Mellado-Gil JM, Mellado-Gil JM, Aguilar-Diosdado M, Aguilar-Diosdado M. High glucose potentiates cytokine- and streptozotocin-induced apoptosis of rat islet cells: effect on apoptosis-related genes. J Endocrinol 2004; 183: 155-162.

32. Plesner A, Soukhatcheva G, Korneluk RG, Verchere CB. XIAP inhibition of $\beta$-cell apoptosis reduces the number of islets required to restore euglycaemia in a syngeneic islet transplantation model. Islets 2010; 2: 18-23.

33. Langhofer M, Hopkinson SB, Jones JC. The matrix secreted by $804 \mathrm{G}$ cells contains lamininrelated components that participate in hemidesmosome assembly in vitro. J Cell Sci 1993; 105 (Part 3): 753-764.

(i) Cell Death and Disease is an open-access journal published by Nature Publishing Group. This work is licensed under a Creative Commons Attribution 4.0 International License. The images or other third party material in this article are included in the article's Creative Commons license, unless indicated otherwise in the credit line; if the material is not included under the Creative Commons license, users will need to obtain permission from the license holder to reproduce the material. To view a copy of this license, visit http://creativecommons.org/licenses/by/4.0/ 